\title{
Generation of a superposition of multiple mesoscopic states of radiation in a resonant cavity
}

\author{
P. K. Pathak* and G. S. Agarwal* \\ Department of Physics, Oklahoma State University, Stillwater, Oklahoma 74078, USA
}

(Received 2 December 2004; published 27 April 2005)

\begin{abstract}
Using resonant interaction between atoms and the field in a high-quality cavity, we show how to generate a superposition of many mesoscopic states of the field. We study the quasiprobability distributions and demonstrate the nonclassicality of the superposition in terms of the zeros of the $Q$ function as well as the negativity of the Wigner function. We discuss the decoherence of the generated superposition state. We propose homodyne techniques of the type developed by [Auffeves et al., Phys. Rev. Lett. 91, 230405 (2003)] to monitor the superposition of many mesoscopic states.
\end{abstract}

DOI: 10.1103/PhysRevA.71.043823

PACS number(s): 42.50.Gy, 32.80.Qk

\section{INTRODUCTION}

The interaction of a single atom with a high-quality cavity has yielded many important results which can be understood in terms of the Jaynes-Cummings model [1]. The advances in this field are extensively reviewed in the literature [2-5]. The generation of a superposition of mesoscopic coherent states has a fundamental place in quantum theory as such a state exhibits quantum interferences and the nonclassical character of the radiation field [6,7]. Eiselt and Risken [8] had discovered that if a cavity contains a coherent field with large photon numbers, say of the order of 10 , then the state of the field for certain times splits into two parts. Each part can be characterized approximately by a coherent state. Several authors have studied many aspects of such splittings $[9,10]$. Auffeves et al. [10] made a successful observation of this splitting. They also devised a novel homodyne method to observe interferences. We note that previously such superpositions were produced using dispersive interactions in a high-quality cavity $[11,12]$ or by using Raman transitions between the center-of-mass degrees of freedom of trapped ions [13].

Earlier studies of the superpositions of more than two coherent states have found many novel features of such states. For example, Zurek [14] noticed that such superpositions lead to structures in phase space which are smaller than Planck's constant. Clearly, we need efficient methods to produce such superpositions. One of the early suggestions [15] for the production of such states was through the passage of a field in a coherent state through a Kerr medium. However, Kerr nonlinearities are usually too small. Another possibility is via the dispersive interaction $[12,16]$ in the cavity. In this paper, we present yet another possibility by using the resonant interaction between the atom and the cavity. We show how successive passage of atoms can be used to produce superpositions involving many coherent states. We specifically concentrate on a superposition of four coherent states.

The organization of the paper is as follows. In Sec. II, we present the details of our proposal to produce a superposition of four coherent states. We examine the Wigner function and

\footnotetext{
*On leave from Physical Research Laboratory, Navrangpura, Ahmedabad-380 009, India.
}

the $Q$ function for such states. We present a comparison of exact and approximate phase-space distribution functions. We further study zeros of the $Q$ function which are a signature of the nonclassical properties of the field. In Sec. III, we show how the passage of the third atom can be used to monitor the superposition of four coherent states. In Sec. IV, we examine the scale over which such a superposition can decohere.

\section{PREPARATION OF A SUPERPOSITION OF FOUR MESOSCOPIC STATES OF THE FIELD}

In a recent experiment, Auffeves et al. [10] have observed a superposition of two distinguishable states of the field in a high-quality cavity using resonant interaction between an atom and the field inside the cavity. This observation is in agreement with the theoretical prediction of Eiselt and Risken [8]. When a two-level Rydberg atom interacts with a microwave field, it splits the field into two parts whose phases move in opposite directions. If the interaction time is chosen such that the phase difference between the split parts becomes $\pi$, then the cavity field can be projected into a superposition similar to a cat state, $|\alpha\rangle+|-\alpha\rangle$.

In this section, we show that the above method can be used for the preparation of a superposition of four mesoscopic states of the field. We consider a two-level Rydberg atom having its higher-energy state $|e\rangle$ and lower-energy state $|g\rangle$ and the cavity has a strong coherent field $|\alpha\rangle$. The atom passes through the cavity and interacts resonantly with the field. The Hamiltonian for the system in the interaction picture is written as

$$
H=\hbar g\left(|e\rangle\left\langle g\left|a+a^{\dagger}\right| g\right\rangle\langle e|\right),
$$

where $g$ is the coupling constant for the atom with the cavity field, and $a\left(a^{\dagger}\right)$ is the annihilation (creation) operator. The state of the atom-cavity system is written as

$$
|\psi(t)\rangle=\sum_{n}\left[c_{e n}(t)|e, n\rangle+c_{g n}(t)|g, n\rangle\right] .
$$

Using Hamiltonian (1), the Schrödinger equation in terms of $c_{e n}$ and $c_{g n}$ is

$$
\dot{c}_{e n-1}=-i g \sqrt{n} c_{g n}
$$




$$
\dot{c}_{g n}=-i g \sqrt{n} c_{e n-1} \text {. }
$$

We assume that the atom enters the cavity in its lower state $|g\rangle$ and after interacting with the field for time $t_{1}$, it is detected in the same state $|g\rangle$. Thus, effectively, the atom absorbs no photon but it projects the cavity field into the state

$$
\begin{gathered}
\left|\psi_{c}\right\rangle=\sum_{n} c_{n} \cos \left(g \sqrt{n} t_{1}\right)|n\rangle, \\
=\frac{1}{2} \sum_{n} c_{n} e^{i g \sqrt{n} t_{1}}|n\rangle+c_{n} e^{-i g \sqrt{n} t_{1}}|n\rangle, \\
c_{n}=\frac{\alpha^{n}}{\sqrt{n !}} e^{-|\alpha|^{2} / 2} .
\end{gathered}
$$

As a result, the cavity field splits into two parts whose phases move in directions opposite to each other. Now we consider the passage of a second identical atom through the cavity. The second atom enters the cavity in its lower state $|g\rangle$ and, after interacting with the field for time $t_{2}$, is detected in the same state $|g\rangle$. The state of the field inside the cavity after passing the second atom is

$$
\begin{aligned}
\left|\psi_{c}^{\prime}\right\rangle= & \sum_{n} c_{n} \cos \left(g \sqrt{n} t_{1}\right) \cos \left(g \sqrt{n} t_{2}\right)|n\rangle \\
= & \frac{1}{4} \sum_{n}\left(c_{n} e^{i g \sqrt{n}\left(t_{1}+t_{2}\right)}|n\rangle+c_{n} e^{-i g \sqrt{n}\left(t_{1}+t_{2}\right)}|n\rangle+c_{n} e^{i g \sqrt{n}\left(t_{1}-t_{2}\right)}|n\rangle\right. \\
& \left.+c_{n} e^{-i g \sqrt{n}\left(t_{1}-t_{2}\right)}|n\rangle\right) .
\end{aligned}
$$

Thus after passing the second atom, the state of the field inside the cavity splits into four parts.

In the coherent state $|\alpha\rangle$, the photon distribution follows Poisson statistics, so in Eq. (8), most of the contribution to the summation comes from the terms $n \approx|\alpha|^{2}$. Thus we can expand $\sqrt{n}$ in phase terms around the average number of photons $\bar{n}=|\alpha|^{2}$ in Eq. (8). In fact for $\bar{n} \sim 10$, only the terms up to second order in $(n-\bar{n})$ are significant and other terms are negligible,

$$
\sqrt{n}=\sqrt{\bar{n}}+\frac{n-\bar{n}}{2 \sqrt{\bar{n}}}-\frac{(n-\bar{n})^{2}}{8 \bar{n}^{3 / 2}} .
$$

If we substitute the value of $\sqrt{n}$ from Eq. (9) in Eq. (8), the term proportional to $n$ will change the phase of the coherent field while the second- and higher-order terms in $(n-\bar{n})$ will distort the shape of the coherent state in phase space. For simplification, in order to understand the nature of the generated superposition state, we do not consider the distortion in the coherent state. Then Eq. (8) can be approximated by

$$
\begin{aligned}
\left|\psi_{c}^{\prime}\right\rangle= & \frac{1}{4}\left[e^{i\left(\eta_{1}+\eta_{2}\right)}\left|\alpha e^{i\left(\theta_{1}+\theta_{2}\right)}\right\rangle+e^{-i\left(\eta_{1}+\eta_{2}\right)}\left|\alpha e^{-i\left(\theta_{1}+\theta_{2}\right)}\right\rangle\right. \\
& \left.+e^{i\left(\eta_{1}-\eta_{2}\right)}\left|\alpha e^{i\left(\theta_{1}-\theta_{2}\right)}\right\rangle+e^{-i\left(\eta_{1}-\eta_{2}\right)}\left|\alpha e^{-i\left(\theta_{1}-\theta_{2}\right)}\right\rangle\right],
\end{aligned}
$$

$$
\eta_{i}=\frac{g t_{i} \sqrt{\bar{n}}}{2}, \quad \theta_{i}=\frac{g t_{i}}{2 \sqrt{\bar{n}}}, \quad i=1,2 .
$$

If we choose interaction times $t_{1}$ and $t_{2}$ such that $\theta_{1}=\pi / 2$ and $\theta_{2}=\pi / 4$, we get the superposition of four mesoscopic coherent states placed in the east, west, north, and south directions in phase space,

$$
\begin{aligned}
\left|\psi_{c}^{\prime}\right\rangle= & \frac{1}{4}\left[e^{-i\left(\eta_{1}-\eta_{2}\right)}\left|\alpha^{\prime}\right\rangle+e^{i\left(\eta_{1}+\eta_{2}\right)}\left|-\alpha^{\prime}\right\rangle+e^{i\left(\eta_{1}-\eta_{2}\right)}\left|i \alpha^{\prime}\right\rangle\right. \\
& \left.+e^{-i\left(\eta_{1}+\eta_{2}\right)}\left|-i \alpha^{\prime}\right\rangle\right] ;
\end{aligned}
$$

where we set $\alpha=\alpha^{\prime} e^{i \pi / 4}$.

Now we calculate the Wigner distribution for the state (7). The Wigner distribution for the state having density matrix $\rho$ can be obtained using coherent states as [17]

$$
W(\gamma)=\frac{2}{\pi^{2}} e^{2|\gamma|^{2}} \int\langle-\beta|\rho| \beta\rangle e^{-2\left(\beta \gamma^{*}-\beta^{*} \gamma\right)} d^{2} \beta .
$$

The density matrix $\rho_{c}$ for state (7) in terms of number states is

$$
\begin{aligned}
\rho_{c}= & \sum_{n, m} \frac{\alpha^{n} \alpha^{* m}}{\sqrt{n ! m !}} e^{-|\alpha|^{2}} \cos \left(g t_{1} \sqrt{n}\right) \cos \left(g t_{2} \sqrt{n}\right) \\
& \times \cos \left(g t_{1} \sqrt{m}\right) \cos \left(g t_{2} \sqrt{m}\right)|n\rangle\langle m| .
\end{aligned}
$$

Using Eqs. (13) and (14), the Wigner distribution for the state (7) is

$$
\begin{aligned}
W(\gamma)= & \frac{2 e^{2|\gamma|^{2}}}{\pi^{2}} \sum_{n, m} \frac{\alpha^{n} \alpha^{* m}}{n ! m !} e^{-|\alpha|^{2}} \cos \left(g t_{1} \sqrt{n}\right) \\
& \times \cos \left(g t_{2} \sqrt{n}\right) \cos \left(g t_{1} \sqrt{m}\right) \cos \left(g t_{2} \sqrt{m}\right) \\
& \times \int\left(-\beta^{*}\right)^{n} \beta^{m} e^{-|\beta|^{2}} \exp \left[-2\left(\beta \gamma^{*}-\beta^{*} \gamma\right)\right] d^{2} \beta .
\end{aligned}
$$

After evaluating the integral, Eq. (15) is simplified to the form

$$
\begin{aligned}
W(\gamma)= & \frac{2 e^{2|\gamma|^{2}}}{\pi} \sum_{n, m} \frac{(-1)^{n+m} \alpha^{n} \alpha^{* m}}{2^{n+m} n ! m !} e^{-|\alpha|^{2}} \cos \left(g t_{1} \sqrt{n}\right) \\
& \times \cos \left(g t_{2} \sqrt{n}\right) \cos \left(g t_{1} \sqrt{m}\right) \cos \left(g t_{2} \sqrt{m}\right) \frac{\partial^{n+m}}{\partial \gamma^{n} \partial \gamma^{* m}} e^{-4|\gamma|^{2}} .
\end{aligned}
$$

In Fig. 1, we show the Wigner distributions for the generated superposition state (8) as well as for the approximated state (10) using some typical values of parameters. There are four patches at the corners corresponding to four mesoscopic states of the field and between each pair of states of the field there are interference fringes indicating the coherence between the states. In the central part, there are sub-Planck structures as noticed by Zurek [14] which form as a result of quantum interference between the two diagonal pairs. The comparison of Figs. 1(a) and 1(b) shows that a significant squeezing perpendicular to the arc of the circle $|z|=|\alpha|$ oc- 
(a)

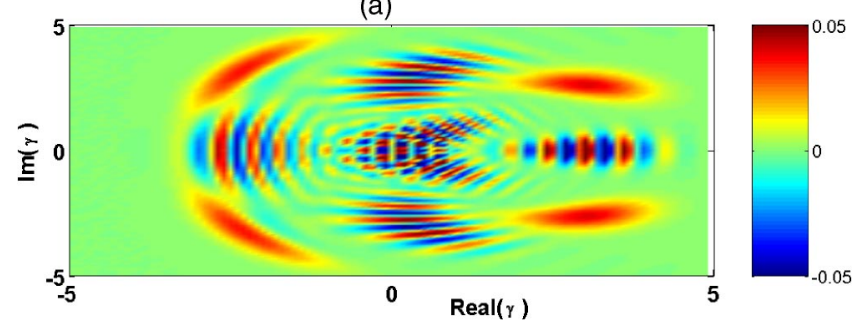

(b)

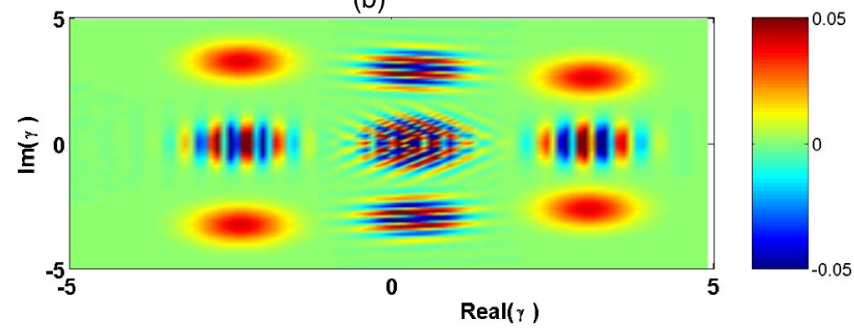

FIG. 1. (Color online) The Wigner distribution $W(\gamma)$ for (a) the generated state (8) and (b) the approximated state (10), using parameters $\alpha=4, g t_{1}=3.7 \pi$, and $g t_{2}=1.9 \pi$.

curs due to the effects of the higher-order terms in $(n-\bar{n})$ [see Eq. (9)]. Squeezing in the resonant Jaynes-Cummings model [18] has been studied very well earlier. As a result of small differences in the field statistics, there are differences in the interference patterns. In Fig. 2, the $Q$ distributions for the states (8) and (10) are shown with the same parameters used in Fig. 1. We select the interaction times such that there is no overlapping between two states of the field. A comparison of Figs. 2(a) and 2(b) shows that the states of the field corresponding to the phases $\pm g \sqrt{n}\left(t_{1}+t_{2}\right)$ [see Eq. (8)] in the
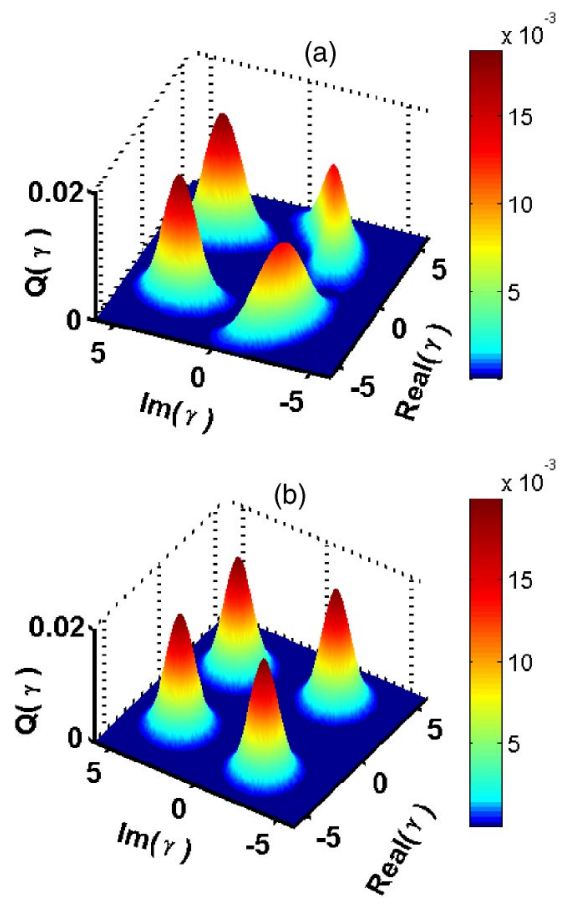

FIG. 2. (Color online) The $Q$-distribution function $Q(\gamma)$ for (a) the generated state (8) and (b) the approximated state (10), using the same parameters as in Fig. 1.

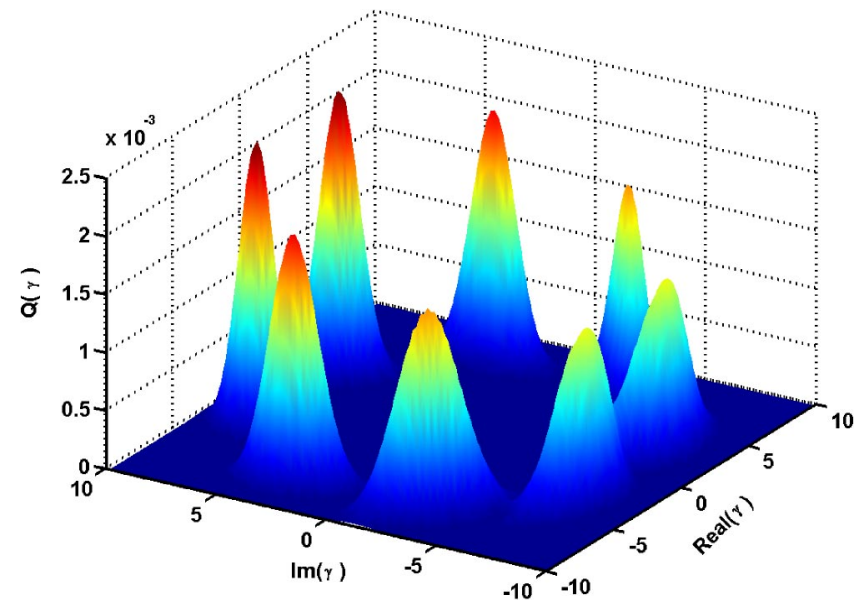

FIG. 3. (Color online) The $Q$-distribution function $Q(\gamma)$ for the generated state after passing three atoms through the cavity, for $\alpha$ $=8$. The interaction times for the first atom, second atom, and the third atom are chosen such that $g t_{1}=8 \pi, g t_{2}=4 \pi$, and $g t_{3}=2 \pi$.

generated mesoscopic state have more spread along the circle $|z|=|\alpha|$ and squeezing perpendicular to it in phase space because of larger distortion terms. Thus the split states of the field in the generated superposition state are situated at the same position as in the approximated state but with changed shape.

We further mention that after passing $N$ atoms through the cavity and properly selecting the interaction times, we can generate the superposition of $2^{N}$ mesoscopic states of the field placed along the arc of a circle of radius $|\alpha|$ in phase space. In Fig. 3, we show the $Q$ distribution for the generated state of the field after passing three atoms through the cavity. It is clear that the generated state is a coherent superposition of eight mesoscopic states.

In this method of preparing superposition of mesoscopic field states, most of the time atoms are in their ground states, thus decoherence effects due to atomic damping are negligible. Only the decoherence of the generated superposition states after passage of the first atom may lead to the generation of an undesirable statistical mixture of states [11]. The mesoscopic states in the generated superposition after passage of the first atom lie on the circle of radii $|\alpha|$ in the phase space. Thus they decohere as $\exp \left(-2|\alpha|^{2} \kappa t\right)$ [cf. Eq. (28)]. The required interaction time for the first atom is given by $g t_{1} \approx \pi|\alpha|$. The required interaction time for the next atom is half the interaction time for the previous atom. We assume that all atoms come in a proper sequence so that the total time in the generation of the state is equal to the total interaction time of the cavity field with the atoms. Then the time required after passage of the first atom in the preparation of $2^{N}$ mesoscopic states, for large $N$, is given by

$$
\frac{t_{1}}{2}+\frac{t_{1}}{4}+\frac{t_{1}}{8}+[(N-1) \text { term }] \approx t_{1} .
$$

Thus the probability of generating the desired state, for large $N$, is reduced by the factor $\exp \left(-2|\alpha|^{2} t_{1} / t_{\text {cav }}\right)$, where $t_{\text {cav }}$ is the lifetime of the field in the cavity. In the case of good cavities, $g t_{c a v} \approx 400$ is feasible. The probability of generating 
state (7) in these cavities, for $|\alpha|^{2} \sim 10$, will be more than $80 \%$.

The relation between the $Q$ distribution and the $P$ distribution for a state is given by

$$
Q(\gamma)=\int P(\alpha) e^{-|\alpha-\gamma|^{2}} d^{2} \alpha .
$$

From Eq. (18), it is clear that for $Q=0$ the $P$ distribution will oscillate between $+v e$ and $-v e$ values. The negative value of $P$ is a signature of the nonclassical nature of the state. Thus the exact zeros of the $Q$ distribution are also signatures of a nonclassical nature. Here it will be interesting to analyze the exact zeros of the $Q$-distribution of the approximated state (12). The $Q$ distribution for state (12) is

$$
\begin{aligned}
Q(\gamma)= & \frac{1}{\pi} \mid\left\langle\gamma \mid \alpha^{\prime}\right\rangle e^{-i\left(\eta_{1}-\eta_{2}\right)}+\left\langle\gamma \mid-\alpha^{\prime}\right\rangle e^{i\left(\eta_{1}+\eta_{2}\right)} \\
& +\left\langle\gamma \mid i \alpha^{\prime}\right\rangle e^{i\left(\eta_{1}-\eta_{2}\right)}+\left.\left\langle\gamma \mid-i \alpha^{\prime}\right\rangle e^{-i\left(\eta_{1}+\eta_{2}\right)}\right|^{2} .
\end{aligned}
$$

The exact zeros of $Q(\gamma)$ will be given by

$$
\begin{aligned}
& \mid\left\langle\gamma \mid \alpha^{\prime}\right\rangle e^{-i\left(\eta_{1}-\eta_{2}\right)}+\left\langle\gamma \mid-\alpha^{\prime}\right\rangle e^{i\left(\eta_{1}+\eta_{2}\right)}+\left\langle\gamma \mid i \alpha^{\prime}\right\rangle e^{i\left(\eta_{1}-\eta_{2}\right)} \\
& \quad+\left\langle\gamma \mid-i \alpha^{\prime}\right\rangle e^{-i\left(\eta_{1}+\eta_{2}\right)} \mid=0 .
\end{aligned}
$$

Thus the $Q$ distribution shows nonclassical behavior at all phase points $\gamma$ satisfying the condition (20). For example, if we take $\alpha^{\prime}$ to be real and observe the $Q$ distribution along the line $\gamma=|\gamma| e^{i \pi / 4}$ in phase space, the condition for nonclassicality (20) simplifies to

$$
\begin{aligned}
& e^{-|\gamma| \alpha^{\prime} / \sqrt{2}} \cos \left[\eta_{1}+\eta_{2}+\frac{|\gamma| \alpha^{\prime}}{\sqrt{2}}\right] \\
& +e^{|\gamma| \alpha^{\prime} / \sqrt{2}} \cos \left[\eta_{1}-\eta_{2}+\frac{|\gamma| \alpha^{\prime}}{\sqrt{2}}\right]=0 .
\end{aligned}
$$

For $|\gamma|=0$ the condition (21) becomes $\cos \eta_{1} \cos \eta_{2}=0$. For $|\gamma| \neq 0$, using the values of $\eta_{1}=\pi\left|\alpha^{\prime}\right|^{2} / 2, \eta_{2}=\pi\left|\alpha^{\prime}\right|^{2} / 4$ [see Eq. (11)], the condition (21) can be rewritten as the simultaneous equations

$$
\begin{gathered}
\frac{|\gamma|}{\sqrt{2} \alpha^{\prime}}+\frac{3 \pi}{4}=\left(2 n_{1}+1\right) \frac{\pi}{2 \alpha^{\prime 2}}, \\
\frac{|\gamma|}{\sqrt{2} \alpha^{\prime}}+\frac{\pi}{4}=\left(2 n_{2}+1\right) \frac{\pi}{2 \alpha^{\prime 2}}, \quad n_{i}=1,2, \ldots .
\end{gathered}
$$

The solution of Eqs. (22) gives $\alpha^{\prime 2}=2\left(n_{1}-n_{2}\right)$, thus $\alpha^{\prime 2}$ must be an even integer and the values of $|\gamma|$ are given by

$$
|\gamma|=\frac{\pi}{2 \sqrt{\left(n_{1}-n_{2}\right)}}\left(3 n_{2}-n_{1}+1\right), \quad n_{1}>n_{2} .
$$

In the above paragraph, we have outlined an analytical approach for getting information about the nonclassical behavior of a state by finding exact zeros of the $Q$ function. It is quite clear from the above that a simple analysis of the $Q$ function can provide information on the nonclassical behavior of the state. Thus this is an alternate analytical approach for checking the nonclassical behavior of the state. In gen- eral, analyzing zeros of the $Q$ function is easier than looking for the -ve value of the Wigner function. In Ref. [19], it is shown that the experiments performed for phase measurement of the radiation field are equivalent to the measurement of the $Q$ function. The measured quantity $w(x, p)$, the probability distribution for the joint measurement of the two quadratures $x$ and $p$, is directly proportional to the $Q$ function. Thus the zeros of the $Q$ function correspond to the minima of the measured quantity $w(x, p)$ in such experiments.

\section{DETECTION OF THE GENERATED SUPERPOSITION OF MESOSCOPIC STATES OF THE FIELD}

In the previous section, we have shown how the cavity field can be projected into a superposition of $2^{N}$ mesoscopic states of the field after passing $N$ atoms through the cavity. The generated state in the cavity can be detected by the conditional probabilities of detection of the atoms used in the preparation itself as the cavity field is entangled with the atomic states. An elegant method can also be homodyne detection [10], which can be implemented in the same experimental setup. After preparing the cavity in the desired superposition state, a resonant external coherent field $|\beta\rangle$ is injected into the cavity. For the sake of simplicity, let us assume that two atoms are passed through the cavity in the preparation of the mesoscopic state of the field [Eq. (7)]. After adding the external field, the state of the resultant field in the cavity is

$$
\begin{aligned}
\left|C_{h}\right\rangle & =\sum_{n} c_{n} \cos \left(g t_{1} \sqrt{n}\right) \cos \left(g t_{2} \sqrt{n}\right) D(\beta)|n\rangle, \\
& =\sum_{m} \sum_{n} c_{n} \cos \left(g t_{1} \sqrt{n}\right) \cos \left(g t_{2} \sqrt{n}\right)\langle m|D(\beta)| n\rangle|m\rangle \\
& =\sum_{m} F_{m}|m\rangle, \\
F_{m} & =\sum_{n} c_{n} \cos \left(g t_{1} \sqrt{n}\right) \cos \left(g t_{2} \sqrt{n}\right)\langle m|D(\beta)| n\rangle,
\end{aligned}
$$

where $D(\beta) \equiv e^{\beta a^{\dagger}-\beta^{*} a}$ is the displacement operator. Now we bring the third atom in its lower-energy state $|g\rangle$ to probe the cavity field. The probability of detecting the probe atom in its lower state $|g\rangle$ after crossing the cavity in time $t_{p}$ is

$$
P_{g}=\sum_{m}\left|F_{m}\right|^{2} \cos ^{2}\left(g t_{p} \sqrt{m}\right) \text {. }
$$

The interaction time $t_{p}$ for the probe atom is selected such that if there are photons in the cavity, it leaves the cavity in its higher-energy state $|e\rangle$ with larger probability. We have shown in the earlier section that all the states of the field in the superposition lie on a circle of radius $|\alpha|$, so if we choose the external field $|\beta\rangle$ having amplitude $|\alpha|$ and phase $\phi$, the probe atom will leave the cavity in its ground state with larger probability when the value of $\pi+\phi$ will match to the phases of the states of the field in the generated superposition. Thus the probability of the probe atom leaving the cav- 


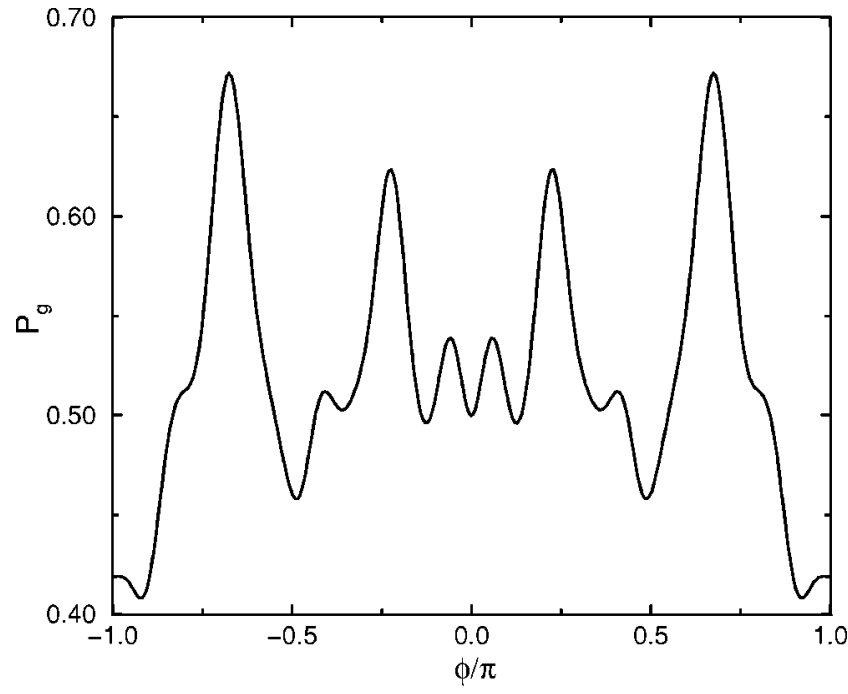

FIG. 4. The probability of detecting a probe atom in its ground state as a function of $\phi$ for the generated superposition (7). The parameters used are the same as in Fig. 1 and the interaction time for the probe atom is selected such that $g t_{p}=1.5 \pi$.

ity in its lower state $|g\rangle$ would, as a function of $\phi$, have peaks corresponding to the positions of the centers of the superposed mesoscopic states. In Fig. 4, we plot the probability of detecting the probe atom in its lower state with $\phi$. It shows four peaks at the positions of the four states of the field in the generated superposition state. The small oscillations in the background are because of the interference effects of residual field components after adding the external field to the cavity.

\section{DECOHERENCE OF THE GENERATED SUPERPOSITION STATE}

Next we study the decoherence of the generated superposition state (8). We are interested in the coherent superposition of four well separated mesoscopic states of the field. The decoherence of such a state will be equivalent to the decoherence of the state (12). This can be done using the master equation

$$
\dot{\rho}=-\frac{\kappa}{2}\left(a^{\dagger} a \rho-2 a \rho a^{\dagger}+\rho a^{\dagger} a\right)
$$

where $\kappa$ is the cavity field decay parameter and we carry an analysis in the absence of thermal photons. For initial state (12), we find the density matrix after time $t$,

$$
\begin{aligned}
\rho(t)= & \frac{1}{16}\left[\left(\left|\alpha_{t}\right\rangle\left\langle\alpha_{t}|+|-\alpha_{t}\right\rangle\left\langle-\alpha_{t}|+| i \alpha_{t}\right\rangle\left\langle i \alpha_{t}|+|-i \alpha_{t}\right\rangle\left\langle-i \alpha_{t}\right|\right)\right. \\
& +e^{-2|\alpha|^{2}\left(1-e^{-\kappa t}\right)}\left(\left|\alpha_{t}\right\rangle\left\langle-\alpha_{t}\left|e^{-2 i \eta_{1}}+\right|-\alpha_{t}\right\rangle\left\langle\alpha_{t}\left|e^{2 i \eta_{1}}+\right| i \alpha_{t}\right\rangle\right. \\
& \times\left\langle-i \alpha_{t}\left|e^{2 i \eta_{1}}+\right|-i \alpha_{t}\right\rangle\left\langle i \alpha_{t}\right| e^{\left.-2 i \eta_{1}\right)}+e^{-|\alpha|^{2}(1-i)\left(1-e^{-\kappa t}\right)}\left(\left|\alpha_{t}\right\rangle\right. \\
& \times\left\langle i \alpha_{t}\left|e^{-2 i\left(\eta_{1}-\eta_{2}\right)}+\right|-i \alpha_{t}\right\rangle\left\langle\alpha_{t}\left|e^{-2 i \eta_{2}}+\right|-\alpha_{t}\right\rangle \\
& \times\left\langle-i \alpha_{t}\left|e^{2 i\left(\eta_{1}+\eta_{2}\right)}+\right| i \alpha_{t}\right\rangle\left\langle-\alpha_{t}\right| e^{\left.-2 i \eta_{2}\right)}+e^{-|\alpha|^{2}(1+i)\left(1-e^{-\kappa t}\right)} \\
& \times\left(\left|i \alpha_{t}\right\rangle\left\langle\alpha_{t}\left|e^{2 i\left(\eta_{1}-\eta_{2}\right)}+\right| \alpha_{t}\right\rangle\left\langle-i \alpha_{t}\left|e^{2 i \eta_{2}}+\right|-i \alpha_{t}\right\rangle\right.
\end{aligned}
$$

(a)

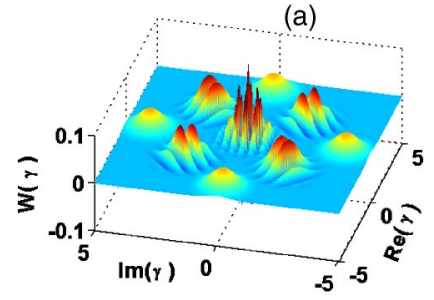

(c)

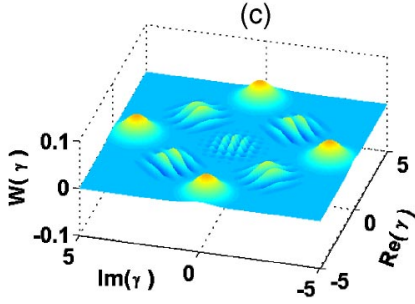

(b)

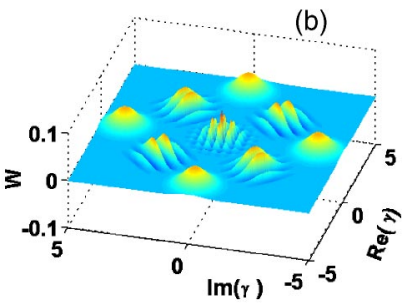

(d)

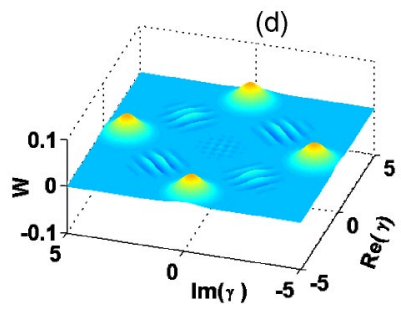

FIG. 5. The decoherence of the approximated state (12) in terms of the Wigner function at different times, (a) for $\kappa t=0$, (b) for $\kappa t$ $=1 / 2|\alpha|^{2}$, (c) for $\kappa t=1 /|\alpha|^{2}$, and (d) for $\kappa t=2 /|\alpha|^{2}$, for $|\alpha|=4$.

$$
\begin{gathered}
\left.\times\left\langle-\alpha_{t}\left|e^{-2 i\left(\eta_{1}+\eta_{2}\right)}+\right|-\alpha_{t}\right\rangle\left\langle i \alpha_{t}\right| e^{\left.2 i \eta_{2}\right)}\right], \\
\alpha_{t}=\alpha^{\prime} e^{-\kappa t / 2} .
\end{gathered}
$$

In Eq. (28), the second, third, and fourth terms reflect the coherent character of the superposition. These are the terms which decohere due to interaction with the environment. The contribution to the Wigner function from the second term in Eq. (28) is

$$
\begin{aligned}
& \frac{e^{-2|\gamma|^{2}-2|\alpha|^{2}\left(1-e^{-\kappa t}\right)}}{4 \pi}\left\{\cos \left[\eta_{1}+i\left(\alpha^{\prime} \gamma^{*}-\alpha^{\prime *} \gamma\right)\right]\right. \\
& \left.+\cos \left[\eta_{1}+\left(\alpha^{\prime} \gamma^{*}+\alpha^{\prime *} \gamma\right)\right]\right\}
\end{aligned}
$$

which decays as $e^{-2|\alpha|^{2}\left(1-e^{-\kappa t}\right)} \approx e^{-2|\alpha|^{2} \kappa t}$ for $\kappa t \ll 1$. This term arises from the coherence between the pair $\left|\alpha^{\prime}\right\rangle,\left|-\alpha^{\prime}\right\rangle$ and the pair $\left|i \alpha^{\prime}\right\rangle,\left|-i \alpha^{\prime}\right\rangle$, and is responsible for the central subPlanck structures. The term in curly bracket can be written as $\left\{\cos \left[\eta_{1}+2 \alpha^{\prime}|\gamma| \sin \theta\right]+\cos \left[\eta_{1}+2 \alpha^{\prime}|\gamma| \cos \theta\right]\right\}$. Thus in any direction $\theta \neq n \pi / 2$ one has an interference pattern which arises from two cosine terms with different periodicity. Thus the sub-Planck structures decohere as $e^{-2|\alpha|^{2} \kappa t}$. The third and the fourth terms in Eq. (28) show the coherence between other pairs of coherent states, and decay as $e^{-|\alpha|^{2} \kappa t}$. In Fig. 5, we plot the decoherence of the approximated state (12) in terms of the Wigner function at different times. As time progresses in Fig. 5 from (a) to (d), the central interference patterns decay faster and disappear earlier than the interference fringes between the coherent states, say $|\alpha\rangle$ and $|i \alpha\rangle$, disappear. This is clear from Eq. (28) that the central interference patterns decohere two times faster than the interference fringes between the coherent states like $|\alpha\rangle$ and $|i \alpha\rangle$.

\section{CONCLUSIONS}

In this paper, we have shown the possibility for generating the superposition of four mesoscopic states of the field using 
resonant interaction between atoms and the field in a cavity. We have discussed the properties of the quasiprobability distributions of the generated state and compared with the superposition of four coherent states. We have discussed the time scale over which the state decoheres and shown that the generated state can be monitored using homodyne detection techniques. Another way to detect such superposition is by doing tomography [20] of such states.
[1] E. T. Jaynes and F. W. Cummings, Proc. IEEE 51, 89 (1963).

[2] H.-I. Yoo and J. H. Eberly, Phys. Rep. 118, 239 (1985).

[3] J. M. Raimond, M. Brune, and S. Haroche Rev. Mod. Phys. 73, 565 (2001).

[4] H. Walther, Adv. At., Mol., Opt. Phys. 32, 379 (1994).

[5] Cavity Quantum Electrodynamics, edited by P. R. Berman (Academic, New York, 1994).

[6] W. P. Schleich, in Quantum Optics in Phase Space (Wiley, New York, 2001).

[7] V. Buzek and P. L. Knight, in Progress in Optics, Vol. XXXIV, edited by E. Wolf (North Holland, Amsterdam, 1995).

[8] J. Eiselt and H. Risken, Opt. Commun. 72, 351 (1989).

[9] J. Gea-Banacloche, Phys. Rev. A 44, 5913 (1991); I. Sh. Averbukh, ibid. 46, R2205 (1992); V. Buzek, H. Moya-Cessa, P. L. Knight, and S. J. D. Phoenix, ibid. 45, 8190 (1992); M. S. Kim and G. S. Agarwal, J. Mod. Opt. 46, 2111 (1999).

[10] A. Auffeves et al., Phys. Rev. Lett. 91, 230405 (2003).
[11] M. Brune et al., Phys. Rev. Lett. 77, 4887 (1996); J. M. Raimond, M. Brune, and S. Haroche, ibid. 79, 1964 (1997).

[12] C. C. Gerry, Phys. Rev. A 53, 3818 (1996).

[13] C. Monroe, D. M. Meekhof, B. E. King, and D. J. Wineland, Science 272, 1131 (1996).

[14] W. H. Zurek, Nature (London) 412, 712 (2001).

[15] K. Tara, G. S. Agarwal, and S. Chaturvedi, Phys. Rev. A 47, 5024 (1993).

[16] G. S. Agarwal and P. K. Pathak, Phys. Rev. A 70, 053813 (2004).

[17] G. S. Agarwal and E. Wolf, Phys. Rev. D 2, 2161 (1970).

[18] J. R. Kuklinski and J. L. Madajczyk, Phys. Rev. A 37, 3175 (1988); R. R. Puri and G. S. Agarwal, ibid. 33, 3610 (1986).

[19] U. Leonhardt and H. Paul, Phys. Rev. A 47, R2460 (1993).

[20] M. S. Kim, G. Antesberger, C. T. Bodendorf, and H. Walther, Phys. Rev. A 58, R65 (1998). 